\section{Il ruolo centrale dell'infermiere nella gestione degli accessi vascolari per la dialisi in un team multidisciplinare}

\section{Caro Direttore,}

ho letto con molto interesse la tua accorata lettera sugli accessi vascolari. In primis mi voglio complimentare per lo stile "neutro" della disamina senza mai cadere nella trappola del karaoke commiserativo. In pratica tu chiedi una riflessione più di testa che di pancia.

Sono convinto che se noi chiedessimo a una persona che ha un minimo di esperienza di organizzazione aziendale farebbe la seguente disamina.

L'organizzazione della nefrologia sul territorio nazionale non ha un "frame" definito di standard clinici di riferimento: come debbono essere fatte determinate procedure; cosa deve avere una struttura complessa di nefrologia per essere definita tale; chi ha titolo a fare cosa perché ha seguito un percorso formativo specifico certificato da un ente super partes e non autoreferenziato, come purtroppo si usa fare nelle nostre "misere cucine".

Questo ha fatto molto comodo a tanti soggetti:

- Ai nefrologi, perché ha lasciato uno spazio infinito alle loro fantasie senza che si preoccupassero dello sviluppo armonico dei servizi che avrebbero dovuto offrire all'utenza.

- Ai politici che hanno usato l'elargizione di titoli per altri "fini" senza nessun collegamento con le necessità epidemiologiche e di programmazione.

- Alla SIN, perché nell'incertezza di riconoscere un titolo a fare qualcosa nel "Centro" anche senza la competenza tecnica specifica del primario, non ha capito l'importanza di porre l'accento sull'accreditamento professionale anche correndo il rischio di pestare i piedi a qualcuno.

Cosi abbiamo reparti che trattano solo gli acuti e altri che trattano i pazienti cronici, reparti che non fanno la peritoneale perché "non ci credono", reparti che non mettono i CVC e reparti che non fanno le fistole, reparti che l'opzione trapianto la vedono remota e reparti (anche in ospedali importanti) che non procurano organi per il trapianto eccetera.

È evidente che se questa "anarchia" organizzativa non ha trovato risposte coerenti nella fase di espansione della nefrologia (diciamo fino ai primi dieci anni del 2000), ora, in un clima di contrazione drammatica delle risorse, è veramente impossibile. Questa è la triste disamina che farebbe un esterno.

$\mathrm{Tu}$, giustamente, poni l'accento sulla diffusione a "macchia di leopardo" della cultura del team multidisciplinare sugli accessi vascolari e hai perfettamente ragione. Ma rimettere questo argomento al centro della formazione del nefrologo clinico mi sembra veramente difficile: in quante scuole di specialità ci sono corsi sugli accessi vascolari e in quante di queste ci sono team dedicati al confezionamento degli accessi vascolari per acuti e cronici?

Corsi e convegni organizzati da un gruppo di sopravvissuti possono sostituirsi alla pratica quotidiana, peraltro sempre più complessa e che richiede competenza tecnica elevata e disponibilità molto superiori rispetto a quando abbiamo cominciato noi? È solo costruendo la mentalità del team (infermiere, nefrologo, chirurgo vascolare, radiologo interventista ecc.), come proponi tu, che forse si potrà superare questa impasse. Ma il perno del team non è il nefrologo o il chirurgo vascolare, io credo debba essere l'infermiere di dialisi. L'infermiere dovrebbe partecipare attivamente nella fase di programmazione della terapia sostitutiva.

Ci dovrebbe essere:

- I'infermiere che segue i pazienti che hanno scelto la dialisi peritoneale;

- l'infermiere che programmerà la costruzione e poi il followup dell'accesso vascolare, per chi farà l'emodialisi;

- I'infermiere che inizierà il percorso del trapianto per gli idonei; eccetera.

Questo presuppone un addestramento completo sulle problematiche tecniche della dialisi e una formazione mirata anche alla gestione dei percorsi assistenziali. Queste problematiche, così come declinate, oggi non sono minimamente sfiorate. Purtroppo, nella maggior parte dei casi, la formazione degli infermieri è limitata alla gestione degli allarmi delle macchine da dialisi. In pochi Centri esiste la cultura degli accessi vascolari e magari un team dedicato che si muove secondo un percorso strutturato.

Sinceramente penso che la SIN potrebbe prevedere dei corsi per colmare questo debito formativo (una Renal week infermieristica), così come dovrebbe dedicare sistematicamente uno spazio infermieristico, nell'ambito del congresso nazionale, a tematiche che coinvolgono competenze multidisciplinari. Quest'anno al congresso nazionale della SIN ci sarà uno spazio per figure professionali diverse, come dietisti e psicologi. Ritengo sarebbe quanto mai opportuno avere sessioni dedicate e cogestite con gli infermieri su tematiche scottanti come gli accessi vascolari, le cure palliative e il fine vita, la gestione della fase predialitica eccetera.

\section{Disclosures}

Financial support: No financial support was received for this submission. Conflict of interest: The author has no conflict of interest.

\section{Giuseppe Rombolà}

Direttore UOC Nefrologia, Ospedale di Circolo e Fondazione Macchi di Varese, Varese

Indirizzo per la corrispondenza:

Giuseppe Rombolà

Direttore UOC Nefrologia

Ospedale di Circolo e Fondazione Macchi di Varese

Via Luigi Borri 57

21100 Varese

giuseppe.rombola@asst-settelaghi.it

Published online: May 15, 2017 